\title{
Intepreting extragalactic backgrounds via angular cross-correlations
}

\section{Marco Regis*}

Dipartimento di Fisica, Università di Torino, and Istituto Nazionale di Fisica Nucleare, Sezione di Torino, via P. Giuria 1, I-10125 Torino, Italy

E-mail: regis@to.infn.it

We discuss methods and results concerning the angular cross-correlation between sky-maps of the extragalactic background radiation in different wavelength bands. The main goal of the study is to extract information on the clustering, redshift distribution and type of the unresolved nonthermal sources, especially at gamma-ray (and radio) frequencies. We describe how this technique can be used to search for "new" populations of sources, including the one possibly provided by annihilation or decay of particle dark matter.

The European Physical Society Conference on High Energy Physics

5-12 July, 2017

Venice

${ }^{*}$ Speaker. 
Let us consider two populations ( $a$ and $b$ ) of astrophysical sources. The probability of finding a pair with an object from population $a$ in a volume $d V_{1}$ of the Universe and an object from population $b$ in $d V_{2}$ is given by [1]: $d P=\rho_{a} d V_{1} \rho_{b} d V_{2}\left(1+\xi_{a b}\left(\vec{r}_{12}\right)\right)$, where $\xi_{a b}$ gives the excess probability as a function of $\vec{r}_{12}$ (i.e., $\xi_{a b}=0$ for Gaussian random fields). On the other hand, we can also write the probability as $d P=\left\langle\rho_{a}\left(\vec{x}_{1}\right) d V_{1} \rho_{b}\left(\vec{x}_{1}+\vec{r}_{12}\right) d V_{2}\right\rangle$, where $\langle.$.$\rangle denotes ensemble average.$ With a few simple steps (and considering $\delta(\vec{x})=\rho(\vec{x}) /\langle\rho\rangle-1$ ), we arrive to the definition of the two-point correlation function:

$$
\xi_{a b}(r)=\left\langle\delta_{a}\left(x_{1}\right), \delta_{b}\left(x_{1}+r\right)\right\rangle_{V}
$$

where we assumed our Universe to satisfy statistical homogeneity (and thus $\xi$ depends only on the modulus of $\vec{r}$ ) and ergodicity (to perform a volume average instead of an ensemble average).

In the works discussed here, we measure the angular cross-correlation between different sky maps and therefore $a$ and $b$ are points in two different maps at different wavelengths. The twopoint cross-correlation function projected on the sky is named $C C F^{(a b)}(\theta)$. It is sometime more convenient to work in the harmonic space (see, e.g., [2]), deriving the cross-correlation angular power spectrum (CAPS) $C_{\ell}^{a b}$. The latter is relate to the $C C F$ via a Legendre transformation:

$$
C C F^{(a b)}(\theta)=\sum_{\ell} \frac{2 \ell+1}{4 \pi} C_{\ell}^{a b} P_{\ell}[\cos (\theta)] .
$$

The general idea is to use maps of known objects (galaxies, galaxy clusters, matter distribution) to isolate contributions from certain populations (in particular, galaxy clusters and particle dark matter) in the unresolved background of maps at different wavelengths.

In Ref. [3], the detection of a cross-correlation signal between Fermi Large Area Telescope diffuse $\gamma$-ray maps and catalogs of clusters was reported. The analysis involved three different catalogs: WHL12, redMaPPer and PlanckSZ. They all show a positive correlation with different amplitudes, see Fig. 1. The signal detection is confirmed by the results of a stacking analysis. The cross-correlation signal extends to rather large angular scales, around 1 degree, that correspond, at the typical redshift of the clusters in these catalogs, to a few to tens of Mpc, i.e. the typical scale-length of the large scale structures in the Universe. Most likely this signal is contributed by the cumulative emission from AGNs associated to the filamentary structures that converge toward the high peaks of the matter density field in which galaxy clusters reside. In addition, our analysis reveals the presence of a second component, more compact in size and compatible with a point-like emission from within individual clusters. At present, we cannot distinguish between the two most likely interpretations for such a signal, i.e. whether it is produced by AGNs inside clusters or if it is a diffuse $\gamma$-ray emission from the intra-cluster medium. This latter, intriguing, hypothesis might be tested by applying this technique to a low redshift large mass cluster sample.

One of the most relevant applications of the cross-correlation analysis is related to the search for particle dark matter (DM). The cross-correlation of gamma-rays with gravitational tracers has been shown to greatly enhance the possibility to infer informations about extragalactic populations with respect to gamma-rays alone (or to other electromagnetic channels) (see, e.g., [2]). One of the most important aspects of this technique is the possibility of performing a tomographic approach (not possible with DM-induced electromagnetic signals alone), using the redshift information associated to the gravitational tracers. The fact that the redshift evolution of the WIMP source is 

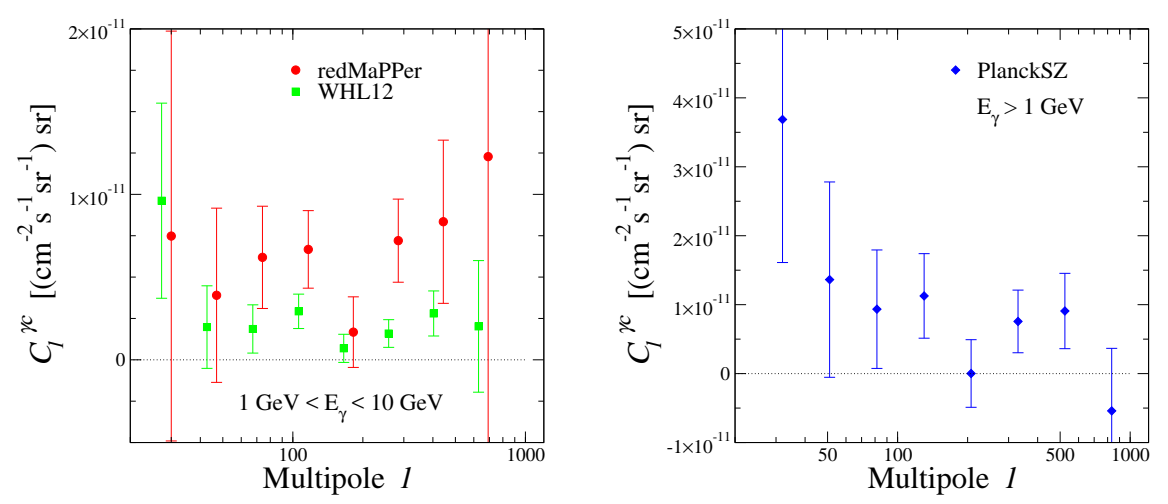

Figure 1: Left panel: Observed CAPS (PSF deconvolved) between the Fermi-LAT $\gamma$-ray map and the redMaPPer (red) and WHL12 (green) catalogs of clusters at $1 \mathrm{GeV}<E_{\gamma}<10 \mathrm{GeV}$. Right panel: Observed CAPS between the Fermi-LAT $\gamma$-ray map at $E_{\gamma}>1 \mathrm{GeV}$ and the PlanckSZ catalog. Figures taken from [3].

predicted to be very different from other astrophysical non-thermal emitters can be used to disentangle a DM signal in these channels.

In Refs. [4, 5], we compare the measured angular cross-correlation between the Fermi-LAT $\gamma$-ray sky and catalogs of extra-galactic objects with the expected signal induced by weakly interacting massive particle (WIMP) DM. We include also a detailed description of the contribution of astrophysical $\gamma$-ray emitters such as blazars, misaligned AGN and star forming galaxies, and perform a global fit to the measured cross-correlation. Five catalogs are considered: SDSS-DR6 quasars, 2MASS galaxies, NVSS radio galaxies, SDSS-DR8 Luminous Red Galaxies and SDSSDR8 main galaxy sample. To model the cross-correlation signal we use the halo occupation distribution formalism to estimate the number of galaxies of a given catalog in DM halos and their spatial correlation properties. The use of different catalogs probing objects at different redshifts reduces significantly, though not completely, the degeneracy among the different $\gamma$-ray components. The presence of a significant WIMP DM signal is allowed by the data but not significantly preferred by the fit, due to a degeneracy with the misaligned AGN component. Assuming modest substructure boost, the analysis excludes thermal annihilation cross sections at 95\% level for WIMP masses up to few tens of GeV. Constraining the low-redshift properties of astrophysical populations with future data will further improve the sensitivity to DM.

Galaxies are a tracers of the DM density in the Universe, but in an indirect way. Indeed, one needs to know the bias between galaxies and the DM density. Weak lensing surveys can instead considered as a direct tracer of the matter density.

In Ref. [6], we measure the cross-correlation between Fermi-LAT gamma-ray photons and over $1000 \mathrm{deg}^{2}$ of weak lensing data from the Canada-France-Hawaii Telescope Lensing Survey (CFHTLenS), the Red Cluster Sequence Lensing Survey (RCSLenS), and the Kilo Degree Survey (KiDS). It is the first measurement of tomographic weak lensing cross-correlations and the first application of spectral binning to cross-correlations between gamma rays and weak lensing. Based on the non-detection of a cross-correlation signal, we derive constraints on WIMP DM excluding the thermal relic cross-section for particle DM masses below $20 \mathrm{GeV}$. 

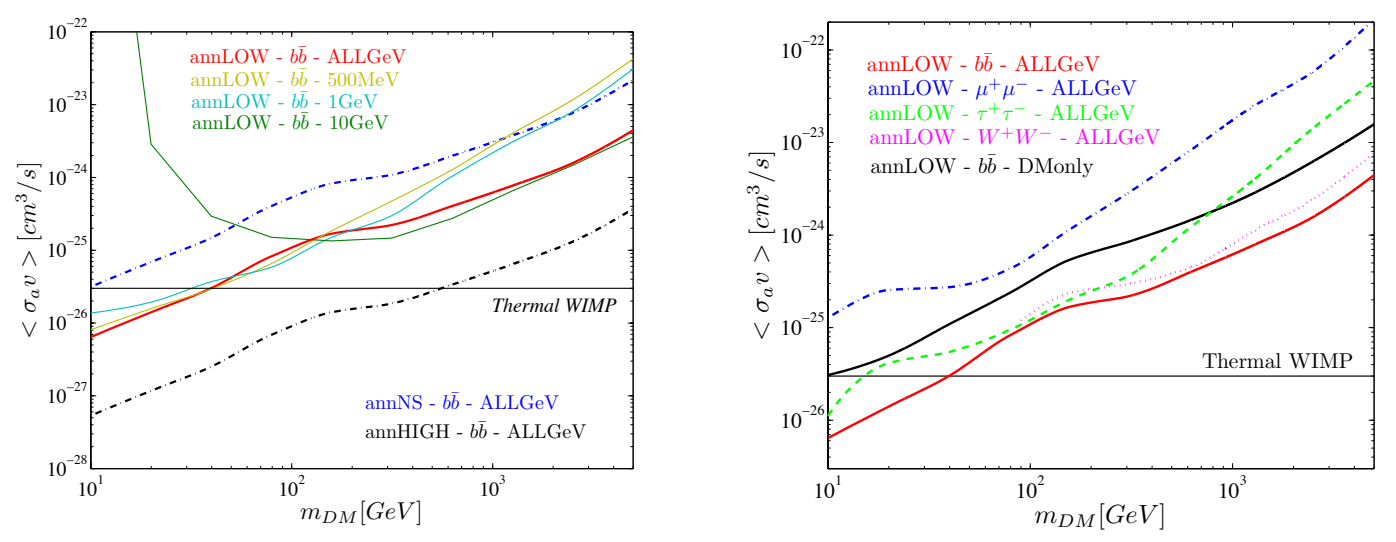

Figure 2: $95 \%$ upper bounds on the DM annihilation rate $\sigma_{a} v$ as a function of the DM mass. Figure taken from [5], to which we refer for details.

WIMP DM can induce a significant extragalactic radiation also in the radio and $\mathrm{X}$ bands. $\mathrm{A}$ multi-wavelength approach to angular auto and cross-correlations has been theoretically discussed in [2]. Next future experiments, like the SKA and its precursors at radio frequencies and e-Rosita and Athena in the X-ray bands, will be able to deeply test the WIMP parameter space.

Going beyond WIMPs, angular correlations have been investigated to study other DM candidates, such as sterile neutrino [7] and axion-like particles [8].

Summarizing, many unknowns/excesses are present in the extragalactic backgrounds at different wavelengths. Are they non-gravitational signature of DM? Angular correlations are a powerful tool to answer this question and test the particle DM hypothesis.

M.R. acknowledges support by the Excellent Young PI Grant: The Particle Dark-matter Quest in the Extragalactic Sky funded by the University of Torino and Compagnia di San Paolo, and by the research grant TAsP (Theoretical Astroparticle Physics) funded by the Istituto Nazionale di Fisica Nucleare (INFN).

\section{References}

[1] Peacock, J. A., Cosmological Physics, Cambridge University Press, 1999, 704.

[2] N. Fornengo and M. Regis, Front. Physics 2 (2014) 6 doi:10.3389/fphy.2014.00006 [arXiv:1312.4835 [astro-ph.CO]].

[3] E. Branchini, S. Camera, A. Cuoco, N. Fornengo, M. Regis, M. Viel and J. Q. Xia, Astrophys. J. Suppl. 228 (2017) no.1, 8 doi:10.3847/1538-4365/228/1/8 [arXiv:1612.05788 [astro-ph.CO]].

[4] M. Regis, J. Q. Xia, A. Cuoco, E. Branchini, N. Fornengo and M. Viel, Phys. Rev. Lett. 114 (2015) no.24, 241301 doi:10.1103/PhysRevLett.114.241301 [arXiv:1503.05922 [astro-ph.CO]].

[5] A. Cuoco, J. Q. Xia, M. Regis, E. Branchini, N. Fornengo and M. Viel, Astrophys. J. Suppl. 221 (2015) no.2, 29 doi:10.1088/0067-0049/221/2/29 [arXiv:1506.01030 [astro-ph.HE]].

[6] T. Troster et al., Mon. Not. Roy. Astron. Soc. 467 (2017) 2706 doi:10.1093/mnras/stx365 [arXiv:1611.03554 [astro-ph.CO]]. 
[7] F. Zandanel, C. Weniger and S. Ando, JCAP 1509 (2015) no.09, 060 doi:10.1088/1475-7516/2015/09/060 [arXiv:1505.07829 [astro-ph.CO]].

[8] Y. Gong, A. Cooray, K. Mitchell-Wynne, X. Chen, M. Zemcov and J. Smidt, Astrophys. J. 825 (2016) no.2, 104 doi:10.3847/0004-637X/825/2/104 [arXiv:1511.01577 [astro-ph.CO]]. 